\title{
Asociación de nivel bajo de PAPP-A en primer trimestre con resultados obstétricos adversos
}

\author{
Álvaro López Soto ${ }^{1}, M^{a}$ Ángeles Jódar Pérez ${ }^{1}$, Olivia García Izquierdo ${ }^{1}$, Mónica Lorente \\ Fernández ${ }^{1}$, Rocío López Pérez ${ }^{1}$, Juan Martínez Uriarte. ${ }^{1}$ \\ ${ }^{1}$ Unidad de Medicina Fetal, Servicio de Obstetricia, Hospital General Universitario Santa Lucía, Cartagena. España.
}

\begin{abstract}
RESUMEN
Antecedentes: La PAPP-A es una proteína utilizada en obstetricia de forma rutinaria para el cribado de aneuploidías de primer trimestre. En los últimos años se está conociendo más acerca de su papel en la función placentaria. Diversos estudios están mostrando una asociación entre un nivel bajo de PAPP-A y distintos eventos obstétricos. Objetivo: Establecer una asociación entre PAPP-A baja y eventos obstétricos adversos. Método: Estudio retrospectivo de casos y controles anidado en una cohorte. Se han recogido las gestaciones únicas con PAPP-A inferior a percentil 5 en primer trimestre durante 2 años. Se ha recogido de la misma cohorte un grupo control, en proporción 2:1. Se compara mediante análisis estadístico la incidencia de eventos obstétricos adversos de cada grupo. Resultados: Se incluyó un total de 285 pacientes en el grupo de casos y 570 pacientes en el grupo control. Se observó un aumento significativo en el grupo de casos de la incidencia de prematuridad, restricción del crecimiento, hipertensión gestacional y diabetes gestacional. Se ha correlacionado la PAPP-A baja con varios eventos obstétricos adversos, incluyendo prematuridad (OR 4,27), diabetes gestacional (OR 2,40), restricción del crecimiento (OR 2,36) e hipertensión gestacional (OR 2,22). No se observó relación con el resto de eventos obstétricos adversos. Conclusión: Un nivel de PAPP-A bajo se asocia con aumentos significativos de prematuridad, diabetes gestacional, restricción del crecimiento e hipertensión gestacional.
\end{abstract}

PALABRAS CLAVE: PAPP-A, función placentaria, eventos obstétricos adversos, prematuridad

\section{SUMMARY}

Background: PAPP-A is a placental protein used in obstetrics as a first trimester marker in aneuploidy screening. In the last few years we are knowing more about its placental function. Some studies are showing a association between low PAPP-A and obstetrical adverse events. Aim: Establish an association between low PAPP-A an obstetrical adverse events. Method: This is a retrospective nested case-control study. We identified each singleton pregnancy with a normal phenotype and a low PAPP-A (under percentile 5) in the last 2 years, and match it with a control group of the same population in a 2:1 proportion. It was compared the incidence of each obstetrical adverse outcomes with statistical analysis. Results: We found 285 patients in the case group and match it with 570 patients from control group. It was observed a significative increase in the incidence of prematurity, intrauterine growth restriction, gestational hypertension and gestational diabetes. A low PAPP-A level was correlated with some obstetrical adverse events, like prematurity (OR 4.27), gestational diabetes (OR 2.40), intrauterine growth restriction (OR 2.36) and gestational hypertension (OR 2.22). We observe no correlation with the rest of outcomes. Conclusions: A low PAPP-A level is related with 
significative increases of prematurity, gestational diabetes, intrauterine growth restriction and gestational hypertension.

\section{KEY WORDS: PAPP-A, placental function, obstetrical adverse events, prematurity}

\section{INTRODUCCIÓN}

La proteína A placentaria asociada al embarazo (PAPP-A) es una glicoproteína secretada por la placenta y que está consolidada desde hace tiempo en la rutina del control gestacional precoz como marcador de aneuploidías (1,2). En 1999 se demostró su papel interactuando con los factores de crecimiento insulínico y su relación con la función placentaria (3). A raíz del estudio de Dugoff y cols (4), se ha puesto en evidencia la relación entre niveles bajos de PAPP-A y peores resultados perinatales, aunque de manera desigual y con alguna controversia (5-13).

El objetivo del presente estudio es investigar la asociación entre bajos valores de PAPP-A séricos en primer trimestre y eventos obstétricos adversos.

\section{PACIENTES Y METODO}

Estudio de casos y controles de tipo retrospectivo. El objetivo era determinar la asociación entre niveles bajos de PAPP-A y eventos obstétricos adversos. La población de estudio fue seleccionada entre todas las gestaciones en el periodo 2013 y 2014 que se realizaron el cribado combinado de aneuploidías en la Unidad Materno Fetal del Hospital General Universitario Santa Lucía, de Cartagena (Murcia) y que mostraron niveles bajos de PAPP-A. Se excluyeron gestaciones múltiples, con patología médica relevante o con fetos con anomalías cromosómicas o malformaciones. Se comparó en una proporción 2:1 con un grupo control de gestantes obtenidas en el mismo periodo y de la misma población, con los mismos criterios de inclusión y con niveles normales de PAPP-A. La investigación fue aprobada por el comité de ética de nuestra institución.

Los niveles séricos de PAPP-A fueron convertidos a valores de múltiplos de la mediana (MoM), mediante la corrección por edad gestacional, etnicidad, hábito tabáquico, peso materno y forma de concepción, usando Automated chemiluminescent immunometric assay (IMMULITE2000® Siemens). Se consideró como PAPP-A baja valores $\leq 0,4$ MoM (percentil 5) y como PAPP-A críticamente baja $\leq 0,2$ MoM. Los eventos obstétricos adversos evaluados fueron la prevalencia de aborto, muerte fetal, prematuridad, retraso de crecimiento, hipertensión gestacional, preeclampsia precoz, preeclampsia tardía, diabetes gestacional y desprendimiento prematuro de placenta.

Se definió aborto a toda pérdida gestacional inferior a 23 semanas de gestación. Muerte fetal se definió como toda pérdida gestacional igual o superior a 23 semanas de gestación. Prematuridad se definió como el parto antes de la semana 37 de gestación. Hipertensión gestacional se definió como la aparición de hipertensión arterial a partir de las 22 semanas de gestación. Se definió el retraso del crecimiento intrauterino como el peso fetal estimado menor de percentil 3. Se definió la preeclampsia precoz como la aparición de hipertensión acompañada de proteinuria antes de las 34 semanas de gestación, y la preeclampsia tardía aquella de aparición posterior a las 34 semanas. Se definió diabetes gestacional como dos o más valores patológicos en el TTOG.

Para el análisis estadístico de los datos se utilizó el software SPSS versión 20.0, utilizándose el test Chi-cuadrado para el análisis de las variables categóricas previamente definidas, considerándose estadísticamente significativo un valor de $p<0,05$, obteniéndose las Odds-Ratio (OR) correspondientes con un $95 \%$ de intervalo de confianza (IC95\%).

\section{RESULTADOS}

Durante el periodo de estudio se identificaron 285 gestantes con una PAPP-A $<0,4 \mathrm{MoM}$ y que cumpliesen criterios de inclusión. Fue seleccionado un grupo control del mismo periodo con una proporción 2:1, con 570 pacientes, con similares características demográficas. De las 855 pacientes incluidas en el estudio, 204 (23,85\%) experimentaron eventos obstétricos adversos. Se observaron diferencias estadísticamente significativas en las variables de prematuridad $(p<0,001)$, restricción del crecimiento intrauterino $(p=0,002)$, hipertensión gestacional $(p=0,044)$ y diabetes gestacional $(p=0,005)$. La Tabla I muestra la incidencia de eventos en ambas series. Un nivel de PAPP-A se correlaciona, por orden decreciente, con un aumento de prematuridad $(\mathrm{OR}=4,27)$, diabetes gestacional $(\mathrm{OR}=2,40)$, restricción del crecimiento intrauterino $(\mathrm{OR}=2,36)$ e hipertensión gestacional $(\mathrm{OR}=2,22)$. La Tabla II muestra el riesgo de eventos obstétricos adversos en mujeres con PAPP-A baja $(<0,4 \mathrm{MoM})$ comparado con el grupo control. 
Tabla I

INCIDENCIA DE EVENTOS OBSTÉTRICOS ADVERSOS EN GRUPOS DE CASOS Y CONTROLES

\begin{tabular}{lccc}
\hline Variables obstétricas & $\begin{array}{c}\text { Controles } \\
(\mathrm{n}=570) \\
\mathrm{n}(\%)\end{array}$ & $\begin{array}{c}\text { Casos } \\
(\mathrm{n}=285) \\
\mathrm{n}(\%)\end{array}$ & Valor $\mathrm{p}$ \\
\hline Aborto & $2(0,35)$ & $3(1,05)$ & 0,222 \\
Muerte fetal & $3(0,52)$ & $4(1,40)$ & 0,195 \\
Prematuridad & $12(2,10)$ & $24(8,42)$ & $<0,001$ \\
Restricción crecimiento & $27(4,73)$ & $30(10,52)$ & 0,002 \\
Enfermedades hipertensivas & $24(4,21)$ & $22(7,71)$ & 0,034 \\
Preeclampsia precoz & $3(0,52)$ & $4(1,40)$ & 0,190 \\
Preeclampsia tardía & $10(1,75)$ & $11(3,85)$ & 0,061 \\
Hipertensión gestacional & $12(2,10)$ & $13(4,56)$ & 0,044 \\
Desprendimiento placenta & $1(0,17)$ & $2(0,70)$ & 0,256 \\
Diabetes gestacional & $23(4,03)$ & $20(7,01)$ & 0,005
\end{tabular}

\section{RIESGO DE EVENTOS OBSTÉTRICOS ADVERSOS EN MUJERES CON PAPP-A BAJA $(<0,4$ MOM) COMPARADO CON EL GRUPO CONTROL}

\begin{tabular}{lcc}
\hline Variables obstétricas & OR & IC95\% \\
\hline Aborto & 3,02 & $0,5020-18,1858$ \\
Muerte fetal & 2,69 & $0,5980-12,1039$ \\
Prematuridad & 4,27 & $2,1060-8,6856$ \\
Restricción de crecimiento fetal & 2,36 & $1,3779-4,0647$ \\
Enfermedades hipertensivas & 1,90 & $1,0476-3,4569$ \\
Preeclampsia precoz & 2,67 & $0,5938-12,0184$ \\
Preeclampsia tardía & 2,24 & $0,9433-5,3588$ \\
Hipertensión gestacional & 2,22 & $1,0007-4,9364$
\end{tabular}

\section{DISCUSIÓN}

La PAPP-A es una glicoproteína descubierta en 1974 por Lin y cols (14), en la sangre de mujeres embarazadas (14). Se trata de un tipo de metaloproteasa producida principalmente en el trofoblasto de la placenta y que aumenta su concentración hasta el final del embarazo $(3,5,11,13,15,16) \mathrm{Su}$ función principal es su acción proteolítica contra la IGFBP (insulin-like growth factor binding protein), permitiendo la liberación del factor de crecimiento insulínico (IGF). Éste juega un importante papel promoviendo la mitosis celular y diferenciación y el control de la invasión trofoblástica de la decidua
$(3,10,13,17)$. Si el nivel de PAPP-A es insuficiente, la IGF permanece en su forma inactiva, Ilevando a un crecimiento fetal y placentario disminuido $(11,18,19)$. Se considera por tanto un buen reflejo de la función placentaria (20-22).

Desde hace más de 20 años la PAPP-A se usa para el cribado de primer trimestre de anomalías cromosómicas. Se sabe que una PAPP-A baja se asocia a trisomías $21,13,18$, y a la monosomía $45 \times 0(11,16,23,24)$. Más recientemente, han surgido trabajos que indican una relación entre niveles bajos de PAPP-A y eventos obstétricos adversos. El trabajo de Dugoff y cols en 2004, usando más de 30.000 pacientes del estudio FASTER, fue de 
los primeros en demostrar asociación de PAPP-A baja con parto pretérmino, hipertensión gestacional, preeclampsia, aborto y bajo peso al nacer (4). Posteriormente han sido varios los trabajos que han asociado PAPP-A baja con aborto, bajo peso al nacer, pequeño para edad gestacional, restricción de crecimiento, parto prematuro, hipertensión gestacional, preeclampsia y/o diabetes gestacional (5-13).

La asociación que más interés ha despertado, ha sido entre la PAPP-A y las enfermedades hipertensivas, en concreto la preeclampsia. Actualmente, hay publicados más de 100 trabajos sobre este tema, la gran mayoría en la última década (25). La implantación embriónica y los procesos de placentación precisan de fenómenos complejos de adaptación a los sistemas maternos cardiovasculares y microvasculares, cuya alteración puede dar lugar a estados hipertensivos como la preeclampsia (13). Dugoff y cols (4), mostraba un OR de 1,79 para el riesgo de preeclampsia, mientras que otros trabajos la han calculado en niveles de 2,2 y 2,5 $(11,16)$. Jelliffe Pawlowski y cols (26), calculó riesgos de preeclampsia precoz 2 veces más altos en multíparas con PAPP-A baja y 3 veces más en nulíparas. Ese riesgo, asociado con una BHCG alta, aumentaba hasta 35 y 11 veces, respectivamente. Nuestro trabajo no mostró una asociación significativa con la preeclampsia precoz ni la tardía, aunque sí con la hipertensión gestacional, con una OR de 2,2.

Existe un gran interés en la creación de un modelo de cribado de preeclampsia de primer trimestre, en el que se incluiría la determinación de la PAPP-A. Usada como único marcador, su sensibilidad y valor predictivo son bastante pobres, llegando como máximo a un VPP del $20 \%(4,27)$. Se han estudiado varios modelos en los que se alcanzaban mayores tasas de detección, combinando marcadores físicos como el doppler de arterias uterinas y otros biomarcadores como PIGF, PP-13, inhibina $A$, pentraxina 3 o selectina- $P(25,28,29)$ No obstante, los actuales modelos se han encontrado inadecuados para su implementación en la práctica clínica (30).

La asociación de PAPP-A baja y pérdida de gestación, ya sea aborto o muerte fetal, también se ha comprobado en varios trabajos. Dugoff y cols (4), concluyeron que una PAPP-A por debajo de percentil 5 se asociaba con un riesgo 5 veces mayor de aborto tardío, siendo corroborado por grupos similares $(4,16,31,32)$ En el caso de una PAPP-A extremadamente baja, por debajo de 0,2 MoM, el riesgo puede llevar a ser casi 20 veces mayor (33). Nuestro trabajo no encontró asociación con niveles de PAPP-A baja y pérdida gestacional. Sí que la encontró con niveles de PAPP-A extremadamente baja, donde el riesgo de aborto fue 21 veces mayor en el grupo de casos.

La asociación entre diabetes gestacional y niveles bajos de PAPP-A está más en discusión. Hay autores que encuentran dicha relación $(34,35)$, mientras que otros no $(16,36,37)$. En el trabajo de Wells y cols (38), el descenso de la PAPP-A fue proporcional a la severidad del defecto metabólico, con niveles progresivamente más bajos a lo largo del espectro de normoglicemia, diabetes gestacional tardía, diabetes gestacional temprana y diabetes gestacional tipo 2. En nuestro caso, sí se encontró dicha asociación con una OR de 2,4.

La asociación entre niveles bajos de PAPP-A y prematuridad ha sido en nuestro trabajo la más fuerte, con el doble de prematuros en el grupo de casos, pese a ser la mitad que el grupo de control. Se ha demostrado una relación entre la edad gestacional media al nacer y la PAPP-A baja $(11,39)$.

El crecimiento fetal parece estar disminuido con niveles bajos de PAPP-A, lo que se refleja en su asociación con restricción del crecimiento, pequeño para edad gestacional y bajo peso al nacer. Hasta hace 20 años se pensaba que el crecimiento en la primera mitad de la gestación dependía únicamente de factores genéticos (7). Hoy día se sabe que influye el desarrollo placentario durante el primer trimestre de la gestación $(13,40)$. Los niveles de PAPP-A baja se han relacionado con la restricción del crecimiento por las mismas causas fisiopatológicas que la preeclampsia (41-43). Gupta y cols (11), mostraron una OR de 7,8 obteniendo nosotros en nuestro trabajo la misma asociación con una OR de 2,36. Los eventos de bajo peso al nacer y pequeño para edad gestacional también están asociados con niveles de PAPP-A baja $(5,15,41,45)$ Poon y cols (45), mostraron que la capacidad predictiva en primer trimestre para pequeño para edad gestacional era del 34\%, pudiendo aumentar al 37\% al combinar la BHCG y la translucencia nucal.

Actualmente está apareciendo una nueva hipótesis respecto a la asociación de niveles altos de PAPP-A (>p99) y macrosomía (13). Ya hay varios trabajos que muestran asociación $(15,38)$, pero en general la literatura es discordante y mixta $(18,37,46,47)$.

Existen otros eventos obstétricos adversos que también se están estudiando, aunque en menor medida. Gupta y cols (11), relacionan la PAPP-A baja con 18 veces más riesgo de oligoamnios; Uccella y cols (48), muestran una relación con el compromiso fetal intraparto y Hancerliogullari y cols (49), lo hacen con la colestasis gestacional.

Pese a la evidencia demostrada, no existen actualmente intervenciones basadas en la evidencia para mejorar los resultados (4,50-52) Destaca 
como pionera la guía clínica de la South Australian Perinatal Practice Guidelines Workgroup (53), en la que se establece el punto de corte en 0,37 MoM (percentil 5) y propone un seguimiento ecográfico adicional para controlar el crecimiento fetal.

\section{CONCLUSIÓN}

Nuestro estudio mostró que un nivel bajo de PAPP-A se asocia con aumentos significativos de prematuridad, diabetes gestacional, restricción del crecimiento e hipertensión gestacional.

\section{REFERENCIAS}

1. Wright D, Syngelaki A, Bradbury I, Akolekar R, Nicolaides $\mathrm{KH}$. First-trimester screening for trisomies 21 , 18 and 13 by ultrasound and biochemical testing. Fetal Diagn Ther. 2014;35(2):118-26.

2. Kagan $\mathrm{KO}$, Wright $\mathrm{D}$, Valencia $\mathrm{C}$, Maiz N, Nicolaides $\mathrm{KH}$. Screening for trisomies 21,18 and 13 by maternal age, fetal nuchal translucency, fetal heart rate, free beta-hCG and pregnancy-associated plasma proteinA. Hum Reprod. 2008;23(9):1968-75.

3. Lawrence JB, Oxvig C, Overgaard MT, Sottrup-Jensen L, Gleich GJ, Hays LG. The insulin-like growth factor (IGF)-dependent IGF binding protein-4 protease secreted by human fibroblasts is pregnancy-associated plasma protein-A. Proc Natl Acad Sci U S A. 1999;96(6):3149-53.

4. Dugoff L, Hobbins JC, Malone FD, Porter TF, Luthy $\mathrm{D}$, Comstock $\mathrm{CH}$, et al. First-trimester maternal serum PAPP-A and free-beta subunit human chorionic gonadotropin concentrations and nuchal translucency are associated with obstetric complications: a populationbased screening study (the FASTER Trial). Am J Obstet Gynecol. 2004;191(4):1446-51.

5. Conserva V, Signaroldi M, Mastroianni C, Stampalija T, Ghisoni L, Ferrazzi E. Distinction between fetal growth restriction and small for gestational age newborn weight enhances the prognostic value of low PAPP-A in the first trimester. Prenat Diagn. 2010;30(10):1007-9.

6. Filippi E, Staughton J, Peregrine E, Jones P, Huttly W, Peebles DM et al. Uterine artery Doppler and adverse pregnancy outcome in women with extreme levels of fetoplacental proteins used for Down syndrome screening. Ultrasound Obstet Gynecol. 2011;37(5):520-7.

7. Smith GC. First trimester origins of fetal growth impairment. Semin Perinatol. 2004;28(1):41-50.

8. Wagner PK, Christians JK. Altered placental expression of PAPPA2 does not affect birth weight in mice. Reprod Biol Endocrinol. 2010;8:90.

9. Gagnon A, Wilson RD, Audibert F, Allen VM, Blight $C$, Brock JA, et al. Obstetrical complications associated with abnormal maternal serum markers analyses. J Obstet Gynaecol Can. 2008;30(10):918-49.

10. Smith GC, Stenhouse EJ, Crossley JA, Aitken DA, Cameron AD, Connor JM. Early pregnancy levels of pregnancy-associated plasma protein a and the risk of intrauterine growth restriction, premature birth, preeclampsia, and stillbirth. J Clin Endocrinol Metab. 2002;87(4):1762-7.
11. Gupta S, Goyal M, Verma D, Sharma A, Bharadwaj $\mathrm{N}$, Kabra M, et al. Adverse pregnancy outcome in patients with low pregnancy-associated plasma proteinA: The Indian Experience. J Obstet Gynaecol Res. 2015;41(7):1003-8.

12. Conover CA, Bale LK, Overgaard MT, Johnstone EW, Laursen UH, Füchtbauer EM. Metalloproteinase pregnancy-associated plasma protein $A$ is a critical growth regulatory factor during fetal development. Development. 2004;131(5):1187-94.

13. Giudice I, Benintende G, Di Nicolò AM, Mangiameli D, Carrara G, Randazzo C, et al. Correlation of neonatal weight with maternal serum levels of pregnancyassociated plasma protein-A during the first trimester of pregnancy: a retrospective study. J Perinat Med. 2015;43(2):227-32.

14. Lin TM, Galbert SP, Kiefer D, Spellacy WN, Gall $S$. Characterization of four human pregnancy-associated plasma proteins. Am J Obstet Gynecol. 1974;118(2):223-36.

15. Cignini $P$, Maggio Savasta LM, Gulino FA, Vitale $S G$, Mangiafico $L$, Mesoraca $A$. Predictive value of pregnancy-associated plasma protein-A (PAPP-A) and free beta-hCG on fetal growth restriction: results of a prospective study. Arch Gynecol Obstet. 2016;293(6):1227-33.

16. Quattrocchi T, Baviera G, Pochiero T, Basile F, Rizzo L, Santamaria A. Maternal serum PAPP-A as an early marker of obstetric complications? Fetal Diagn Ther. 2015;37(1):33-6.

17. Irwin JC, Suen LF, Martina NA, Mark SP, Giudice LC. Role of the IGF system in trophoblast invasion and pre-eclampsia. Hum Reprod. 1999;14 Suppl 2:90-6.

18. Peterson SE, Simhan HN. First-trimester pregnancyassociated plasma protein $A$ and subsequent $a b$ normalities of fetal growth. Am J Obstet Gynecol. 2008;198(5):e43-5.

19. Costa SL, Proctor L, Dodd JM, Toal M, Okun N, Johnson JA, et al. Screening for placental insufficiency in high-risk pregnancies: is earlier better? Placenta. 2008;29(12):1034-40.

20. Odibo AO, Zhong Y, Longtine M, Tuuli M, Odibo L, Cahill AG. First-trimester serum analytes, biophysical tests and the association with pathological morphometry in the placenta of pregnancies with preeclampsia and fetal growth restriction. Placenta. 2011 Apr;32(4):333-8.

21. Rizzo G, Silvestri E, Capponi A, Servadei F, Pietrolucci ME, Capece A, et al. Histomorphometric characteristics of first trimester chorionic villi in pregnancies with low serum pregnancy-associated plasma proteinA levels: relationship with placental three-dimensional power doppler ultrasonographic vascularization. J Matern Fetal Neonatal Med. 2011;24(2):253-7.

22. Metzenbauer M, Hafner E, Schuchter K, Philipp K. First-trimester placental volume as a marker for chromosomal anomalies: preliminary results from an unselected population. Ultrasound Obstet Gynecol. 2002;19(3):240-2.

23. Wald NJ, Kennard A, Hackshaw AK. First trimester serum screening for Down's syndrome. Prenat Diagn. 1995;15(13):1227-40.

24. Bindra R, Heath V, Liao A, Spencer K, Nicolaides KH. One-stop clinic for assessment of risk for trisomy 21 at 
11-14 weeks: a prospective study of 15030 pregnancies. Ultrasound Obstet Gynecol. 2002;20(3):219-25.

25. Kalousová M, Muravská A, Zima T. Pregnancy-associated plasma protein A (PAPP-A) and preeclampsia. Adv Clin Chem. 2014;63:169-209.

26. Jelliffe-Pawlowski LL, Baer RJ, Currier RJ, Lyell DJ, Blumenfeld YJ, El-Sayed YY, et al. Early-onset severe preeclampsia by first trimester pregnancy-associated plasma protein $\mathrm{A}$ and total human chorionic gonadotropin. Am J Perinatol. 2015;32(7):703-12.

27. Poon LC, Kametas NA, Maiz N, Akolekar R, Nicolaides $\mathrm{KH}$. First-trimester prediction of hypertensive disorders in pregnancy. Hypertension. 2009;53(5):8128.

28. Akolekar R, Syngelaki A, Sarquis R, Zvanca M, Nicolaides $\mathrm{KH}$. Prediction of early, intermediate and late pre-eclampsia from maternal factors, biophysical and biochemical markers at 11-13 weeks. Prenat Diagn. 2011;31(1):66-74.

29. Spencer K, Yu CK, Cowans NJ, Otigbah C, Nicolaides $\mathrm{KH}$. Prediction of pregnancy complications by first-trimester maternal serum PAPP-A and free beta-hCG and with second-trimester uterine artery Doppler. Prenat Diagn. 2005;25(10):949-53.

30. American College of Obstetricians and Gynecologists; Task Force on Hypertension in Pregnancy. Hypertension in pregnancy. Report of the American College of Obstetricians and Gynecologists' Task Force on Hypertension in Pregnancy. Obstet Gynecol. 2013;122(5):1122-31.

31. Spencer K, Cowans NJ, Avgidou K, Nicolaides KH. First-trimester ultrasound and biochemical markers of aneuploidy and the prediction of impending fetal death. Ultrasound Obstet Gynecol. 2006;28(5):637-43.

32. Tong S, Ngian GL, Onwude JL, Permezel M, Saglam B, Hay $S$ et al. Diagnostic accuracy of maternal serum macrophage inhibitory cytokine-1 and pregnancy-associated plasma protein-A at 6-10 weeks of gestation to predict miscarriage. Obstet Gynecol. 2012;119(5):1000-8.

33. Karim JN, Sau A. Low pregnancy associated plasma protein- $A$ in the 1st trimester: is it a predictor of poor perinatal outcome? J Obstet Gynaecol. 2013;33(4):351-4.

34. Beneventi F, Simonetta M, Lovati E, Albonico G, TineIli $C$, Locatelli $E$, et al. First trimester pregnancy-associated plasma protein-A in pregnancies complicated by subsequent gestational diabetes. Prenat Diagn. 2011;31(6):523-8.

35. Ong CY, Liao AW, Spencer K, Munim S, Nicolaides $\mathrm{KH}$. First trimester maternal serum free beta human chorionic gonadotrophin and pregnancy associated plasma protein $A$ as predictors of pregnancy complications. BJOG. 2000;107(10):1265-70.

36. Savvidou MD, Syngelaki A, Muhaisen M, Emelyanenko $\mathrm{E}$, Nicolaides $\mathrm{KH}$. First trimester maternal serum free B-human chorionic gonadotropin and pregnancy-associated plasma protein $A$ in pregnancies complicated by diabetes mellitus. BJOG. 2012;119(4):410-6.

37. Tul N, Pusenjak S, Osredkar J, Spencer K, NovakAntolic Z. Predicting complications of pregnancy with first-trimester maternal serum free-betahCG, PAPP-A and inhibin-A. Prenat Diagn. 2003;23(12):990-6.
38. Wells G, Bleicher K, Han X, McShane M, Chan YF, Bartlett A. Maternal Diabetes, Large-for-GestationalAge Births, and First Trimester Pregnancy-Associated Plasma Protein-A. J Clin Endocrinol Metab. 2015;100(6):2372-9.

39. Akolekar R, Syngelaki A, Poon L, Wright D, Nicolaides $\mathrm{KH}$. Competing risks model in early screening for preeclampsia by biophysical and biochemical markers. Fetal Diagn Ther. 2013;33(1):8-15.

40. Poon LC, Zaragoza E, Akolekar R, Anagnostopoulos $\mathrm{E}$, Nicolaides $\mathrm{KH}$. Maternal serum placental growth factor (PIGF) in small for gestational age pregnancy at $11(+0)$ to $13(+6)$ weeks of gestation. Prenat Diagn. 2008;28(12):1110-5.

41. Huynh L, Kingdom J, Akhtar S. Low pregnancy-associated plasma protein A level in the first trimester. Can Fam Physician. 2014;60(10):899-903.

42. Kirkegaard I, Uldbjerg N, Henriksen TB. PAPP-A and free $\mathrm{B}-\mathrm{hCG}$ in relation to admission to neonatal intensive care unit and neonatal disease. Prenat Diagn. 2011;31(12):1169-75.

43. Krantz D, Goetzl L, Simpson JL, Thom E, Zachary J, Hallahan TW. Association of extreme first-trimester free human chorionic gonadotropin-beta, pregnancyassociated plasma protein $A$, and nuchal translucency with intrauterine growth restriction and other adverse pregnancy outcomes. Am J Obstet Gynecol. 2004;191(4):1452-8.

44. Carbone JF, Tuuli MG, Bradshaw R, Liebsch J, Odibo $A O$. Efficiency of first-trimester growth restriction and low pregnancy-associated plasma protein-A in predicting small for gestational age at delivery. Prenat Diagn. 2012;32(8):724-9.

45. Poon LC, Karagiannis G, Staboulidou I, Shafiei A, Nicolaides $\mathrm{KH}$. Reference range of birth weight with gestation and first-trimester prediction of small-forgestation neonates. Prenat Diagn. 2011;31(1):58-65.

46. Plasencia W, González Dávila E, Tetilla V, Padrón Pérez E, García Hernández JA, González González NL. First-trimester screening for large-for-gestational-age infants. Ultrasound Obstet Gynecol. 2012;39(4):38995.

47. Pihl K, Sørensen TL, Nørgaard-Pedersen B, Larsen SO, Nguyen TH, Krebs L et al. First-trimester combined screening for Down syndrome: prediction of low birth weight, small for gestational age and pre-term delivery in a cohort of non-selected women. Prenat Diagn. 2008;28(3):247-53.

48. Uccella S, Colombo GF, Bulgheroni CM, Serati M, Bogani G, Salvatore S. First-trimester maternal serum screening and the risk for fetal distress during labor. Am J Obstet Gynecol. 2009 Aug;201(2):166.e1-6.

49. Hançerlioğullari N, Aktulay A, Engin-Üstün Y, Ozkan MŞ, Oksuzoglu A, Danişman N. Pregnancy-associated plasma protein a levels are decreased in obstetric cholestasis. Clin Exp Obstet Gynecol. 2015;42(5):6178.

50. Dugoff L; Society for Maternal-Fetal Medicine. Firstand second-trimester maternal serum markers for aneuploidy and adverse obstetric outcomes. Obstet Gynecol. 2010;115(5):1052-61.

51. Gagnon A, Wilson RD, Audibert F, Allen VM, Blight $C$, Brock JA, et al. Obstetrical complications associated 
with abnormal maternal serum markers analytes. J Obstet Gynaecol Can. 2008;30(10):918-49.

52. Razavi AS, Chasen ST. Low PAPP-A: the impact of ultrasound to evaluate fetal growth. Prenat Diagn. 2016;36(2):112-6.
53. Management of women with a low PAPP-A and normal chromosomes. South Australian Perinatal Practice Guideline CG152. Department of Health, Government of South Australia. 